\title{
Conditional Maximum Likelihood Estimation in Probability-Branched Multistage Designs
}

\author{
Jan Steinfeld (iD ${ }^{1,2, *}$ \& Alexander Robitzsch (iD ${ }^{3,4}$ \\ ${ }^{1}$ University of Vienna \\ 2 Austrian Federal Ministry of Education, Science and Research \\ ${ }^{3}$ IPN - Leibniz Institute for Science and Mathematics Education, Kiel, Germany \\ ${ }^{4}$ Centre for International Student Assessment (ZIB), Kiel, Germany
}

\begin{abstract}
This article introduces conditional maximum likelihood (CML) item parameter estimation in multistage designs based on probabilities $p(j)$ for choosing a particular path conditional on a raw score $x_{+}$in a previous module. This type of multistage design is applied to ensure a minimum exposure rate for all items, for example in international large-scale assessments (ILSAs). For the item parameter estimation, various likelihood-based methods are available. While the marginal maximum likelihood method (MML) provides consistent estimates in multistage designs, the CML method in its original formulation leads to biased item parameter estimates. Zwitser and Maris (2015, Psychometrika) proposed a modified CML estimation for multistage designs with deterministic routing rules that provide practically unbiased item parameter estimates. First, this approach is illustrated for sequential and cumulative multistage designs. Subsequently, another modification of the CML estimation method is introduced, which is necessary for multistage designs with probabilistic routing. In a simulation study, it is shown that this modified CML estimation method provides also in probabilistic multistage designs practically unbiased item parameter estimates.
\end{abstract}

Keywords: multistage testing; conditional maximum likelihood; Rasch model; parameter estimation; probability-based multistage testing

\section{Introduction}

Computer-based assessment (CBA) possesses several organizational, technical, and psychometric advantages. CBA is characterized as more economical, the data are available

\footnotetext{
* Correspondence should be sent to: Jan Steinfeld, University of Vienna, Faculty of Psychology, Department of Developmental and Educational Psychology, Differential Psychology and Psychological Assessment, Liebiggasse 5, A-1010 Vienna, Austria. Email: jan.steinfeld@univie.ac.at
} 
more quickly (see, e.g., Organisation for Economic Co-operation and Development [OECD], 2010), more flexibility in test administration is guaranteed, and it contributes to improving the test experience of the participants (OECD, 2010). All these advantages may have led to the increasing use of CBA also in international large-scale assessments (ILSAs), as well as in educational measurement in general. In the last decade, several ILSAs switched to more or less CBA such as Programme for International Student Assessment (PISA; OECD, 2019a), Programme for the International Assessment of Adult Competencies (PIAAC; OECD, 2019b), the National Assessment of Educational Progress (NAEP; Campbell, Hombo, \& Mazzeo, 2000; Zhang, Xie, Park, Kim, Broer, \& Bohrnstedt, 2016), Trends in the International Mathematics and Science Study (collection cycle 2019 on computer-based assessment system; eTIMSS; TIMSS; Fishbein, Martin, Mullis, \& Foy, 2018), or Progress in International Reading Literacy Study (PIRLS; Mullis \& Martin, 2019). While paper-based assessments are more or less tied to linear fixed-length tests (LFTs; see, e.g., Magis, Yan, \& von Davier, 2017; van der Linden, 2005), there are only a few examples of paper-based adaptive testing in the field of psychological assessment (see Cronbach \& Gleser, 1957; Kubinger \& HolocherErtl, 2014, for some of those exceptions). CBA allows more sophisticated item selection techniques such as multistage testing (MST; Drasgow, Luecht, \& Bennett, 2006; Lord, 1971; Lord, Novick, \& Birnbaum, 1968; Luecht \& Nungester, 1998; Magis et al., 2017; Zenisky, Hambleton, \& Luecht, 2009) or computerized adaptive testing (CAT; Lord, 1980; van der Linden \& Glas, 2010; Wainer et al., 2000; Weiss, 1983). Compared to LFTs, adaptive test designs are usually more efficient in terms of test length, providing the same or even higher measurement precision, especially for very high and low performing persons (Betz \& Weiss, 1974; Chang, 2015; Cronbach \& Gleser, 1957; Hendrickson, 2007; Jodoin, Zenisky, \& Hambleton, 2006; Kim \& Plake, 1993; Lord, 1971, 1980; van der Linden \& Glas, 2010; Wainer et al., 2000; Weiss, 1982, 1983; Weiss \& Kingsbury, 1984). The efficiency might probably also led to its popularity in ILSAs (e.g., Chang, 2015), some assessments applied MST designs like PISA, PIAAC, eTIMSS, NAEP, or PIRLS. For the estimation of the item parameters, various methods are available. While the marginal maximum likelihood (MML) estimation method in MST designs is applicable without leading to biased item parameter estimates (Eggen \& Verhelst, 2011; Glas, 1988; Wang, Chen, \& Jiang, 2019), conditional maximum likelihood (CML) based parameter estimation in MST is only feasible by modifying the CML estimation method as proposed by Zwitser and Maris (2015). More precisely this modification is applicable for deterministic MST, while probabilistic MST as used in ILSAs needs further modification of the estimation process in CML estimation which will be introduced in the following. First, MST is presented in general and routing rules are outlined. Subsequently, item parameter estimation in deterministic MST is discussed and the modification for probabilistic-based MST is introduced. 


\subsection{Multistage Tests}

In MST, it is necessary to define groups of items, called modules in advance of test administration. Each of these modules has a particular targeted mean difficulty and a specified variance of difficulties. Other additional content-related criteria, for example, the ratio of response formats within modules or similar content stimuli, are conceivable. A simple MST design has at least three modules that differ in its mean difficulty, two paths, and two stages (see Figure 1a).

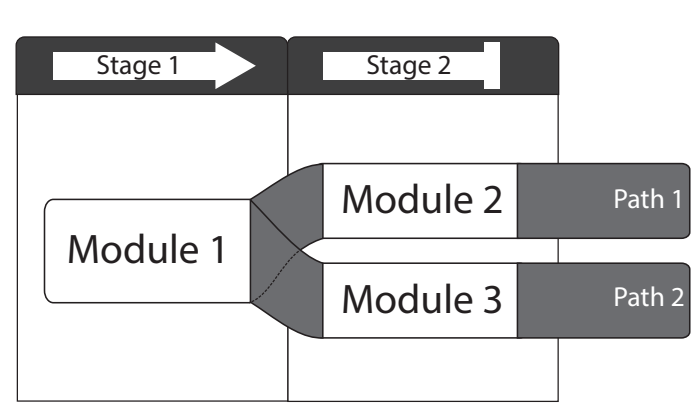

(a) Multistage design with three modules, two stages, and two paths.

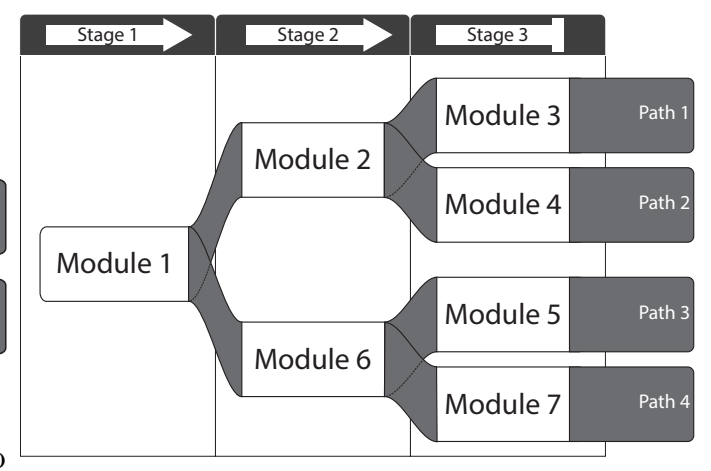

(b) Multistage design with seven modules, three stages, and four paths.

Figure 1. Two examples of multistage designs

Depending on the respective performance and, if available, additional performancerelated prior information together with the routing module, each test person receives further modules that best correspond to the currently estimated ability. The provisional ability of a person is determined given the item responses on processed modules (e.g., number of solved items Lord, 1980). In each of the stages, there are usually at least two modules that differ in their mean difficulty. After every module, the persons' ability is updated, and the next module is chosen. High-performing persons receive those modules that have on average higher mean difficulty, and low-performing persons receive modules with lower mean difficulty. A deviation from this strategy could always be appropriate if it is to be feared that, for example, responses for particularly difficult respective easy modules might be underrepresented to fulfill requirements on minimal item exposure. Svetina, Liaw, Rutkowski, and Rutkowski (2019) stated that it is particularly crucial in ILSAs to control item exposure rate to ensure a stable estimation of item parameters (see also Chen, Yamamoto, \& von Davier, 2014; Yamamoto \& Khorramdel, 2018). One strategy for such control of item exposure rate with additional criteria for the routing process is introduced in PIAAC (Chen et al., 2014; Yamamoto \& Khorramdel, 2018; Yamamoto, Shin, \& Khorramdel, 2018) and PISA (Yamamoto, Shin, \& Khorramdel, 2019) and referred to in the following as probabilistic routing which will be outlined in the following. 
1.1.1 Routing Strategies. Choosing the appropriate MST routing strategies also influences the efficiency, and precision of the item parameter estimation (Lord, 1980). The routing algorithm for assigning a module to a person is, therefore, a significant factor for the efficiency of an MST design. Different types of routing rules have been proposed in the literature. The number of solved items in the current module, in the following, referred to as number-correct score (NC), might be the simplest routing rule (see also Lord, 1980; Zenisky et al., 2009). Persons reaching test score $j$ are assigned at a given cut score of $c$, with $j \leq c$, to an easier module. Several variations of this procedure are conceivable like the inclusion of the information of all previously administered modules. In the following, this type of routing is referred to as cumulative number-correct score (cNC) (Kim, Moses, \& Yoo, 2015; Svetina et al., 2019). During MST administration, increasingly information about the person's ability is available which results in a more accurate estimate of the ability providing a more accurate routing. Especially in ILSAs, the design has to be optimized for estimation purposes to ensure a minimum (sufficient) amount of item responses to ensure stable item parameter estimation. For this, next to the described deterministic routing strategy based on the $\mathrm{NC}$ or $\mathrm{cNC}$ score, an additional probabilistic criterion is introduced. For each raw score, probabilities are defined that affect the deterministic routing process only to a probability $p(j)$ to the optimal module and with a probability $1-p(j)$ into an easier or more difficult module. This type of routing ensures that also very easy and difficult modules are administered to a sufficient amount for reliable item parameter estimation. In the following, the $\mathrm{NC}$ and $\mathrm{cNC}$ routing are referred to as deterministic routing strategies in contrast to the probabilistic routing (see also Section 4).

\section{Parameter Estimation}

Various methods are available to fit an item response theory model such as the Rasch model (1PL; Rasch, 1960) on data obtained by MST designs to estimate item parameters (see, e.g., Steinfeld \& Robitzsch, 2021). While persons are either treated as random or fixed, item parameters are typically regarded as fixed (see, e.g., De Boeck, 2008; Holland, 1990; Molenaar, 1995; San Martin \& De Boeck, 2015, for a further discussion on this topic). The estimation methods considered in the following are all based on maximum likelihood estimation. Some of the most commonly used likelihood-based methods are the marginal maximum likelihood (MML) and the conditional maximum likelihood (CML) method, which is briefly discussed to describe the estimation in MST designs in more detail later (see, e.g., Baker \& Kim, 2004; Fischer, 2007). In the following, we consider dichotomous item responses and utilized the $1 \mathrm{PL}$ model.

Let $X_{p i} \in\{0,1\}$ denoting an independent Bernoulli item response variable with the observation $x_{p i}$ of person $p ; p=1,2, \ldots, P$ on item $i ; i=1,2, \ldots, I$, item difficulties 
$\boldsymbol{\beta}=\left(\beta_{1}, \beta_{2}, \ldots, \beta_{I}\right)$ and ability $\boldsymbol{\theta}=\left(\theta_{1}, \theta_{2}, \ldots, \theta_{P}\right)$. Assuming local stochastic independence, the probability $P\left(\mathbf{x}_{p} \mid \theta_{p}, \boldsymbol{\beta}\right)$ of the response vector $\mathbf{x}_{p}$ of person $p$ can be expressed as:

$$
P\left(\mathbf{X}_{p}=\mathbf{x}_{p} \mid \theta_{p}, \boldsymbol{\beta}\right)=\frac{\exp \left(x_{p+} \theta_{p}-\sum_{i=1}^{I} x_{p i} \beta_{i}\right)}{\prod_{i=1}^{I}\left(1+\exp \left(\theta_{p}-\beta_{i}\right)\right)},
$$

here $x_{p+}=\sum_{i=1}^{I} x_{p i}$ denotes the observed raw score of person $p$. There are $\left(\begin{array}{c}I \\ x_{p+}\end{array}\right)$ different combinations to obtain raw score $x_{+}$satisfying $\sum_{i=1}^{I} x_{p i}=x_{+}$in a data set with $I$ items. Therefore following Equation (1) the response vector $\mathbf{x}_{p}=\left(x_{p 1}, x_{p 2}, \ldots, x_{p I}\right)$ with given ability $\theta_{p}$ of person $p$ and item difficulties $\boldsymbol{\beta}$ can be expressed as:

$$
P\left(\mathbf{x}_{p}=x_{p+} \mid \theta_{p}, \boldsymbol{\beta}\right)=\frac{\exp \left(x_{p+} \theta_{p}\right) \gamma_{x_{p+}}}{\prod_{i=1}^{I}\left(1+\exp \left(\theta_{p}-\beta_{i}\right)\right)}
$$

with $\gamma_{x_{p+}}$ as the basic elementary symmetric function of order $x_{p+}$ of $\beta_{1}, \beta_{2}, \ldots, \beta_{I}$ and $\gamma_{x_{p+}}=\sum_{\mathbf{x}_{p i}}^{x_{p+}} \exp \left(-\sum_{i=1}^{I} x_{p i} \beta_{i}\right)=\gamma\left(x_{p+} ; \beta_{1}, \beta_{2}, \ldots, \beta_{I}\right)$ expressing the different combinations of the raw score $x_{p+}$. The likelihood for the response matrix $\mathbf{X}$ can be expressed as:

$$
L(\mathbf{X} \mid \boldsymbol{\theta}, \boldsymbol{\beta})=\frac{\exp \left(\sum_{p=1}^{P} x_{p+} \theta_{p}-\sum_{p=1}^{P} \sum_{i=1}^{I} x_{p i} \beta_{i}\right)}{\prod_{p=1}^{P} \prod_{i=1}^{I}\left(1+\exp \left(\theta_{p}-\beta_{i}\right)\right)}
$$

In the following, two different estimation methods for Equation (3) are introduced.

\subsection{Marginal Maximum Likelihood estimation}

A very popular approach of item parameter estimation is the MML method (Bock \& Aitkin, 1981; Bock \& Lieberman, 1970; Thissen, 1982). Considering the likelihood function in Equation (3), in the MML case, the likelihood for the observed data matrix $\mathbf{X}$ is the product of the integrals of the respective likelihood of the response patterns and can be expressed as:

$$
L_{M M L}\left(\mathbf{X} \mid \boldsymbol{\beta}, \mu, \sigma^{2}\right)=\prod_{x_{+}=0}^{I}\left[\int \frac{\exp \left(x_{+} \theta-\sum_{i=1}^{I} s_{i} \beta_{i}\right)}{\prod_{i=1}^{I}\left(1+\exp \left(\theta-\beta_{i}\right)\right)} \mathrm{g}(\theta ; \boldsymbol{\alpha}) d \theta\right]^{n_{x_{+}}}
$$

with $s_{i}=\sum_{p=1}^{P} x_{p i}$ the item score of item $i, n_{x_{+}}$as the number of test persons with the raw score $x_{+}$. For the parametric case, a distribution $\mathrm{G}$ with probability density function $\mathrm{g}(\theta ; \boldsymbol{\alpha})$ and $\alpha$ defining the parameters of the latent ability distribution for the person parameter $\theta$ is introduced. It is assumed, that the persons are a random sample from a population e.g., $\theta \sim \mathrm{N}\left(\mu, \sigma^{2}\right)$ thus the random variable $\theta$ is integrated out of the marginal log-likelihood function. For model identification purposes, if a normal distribution is assumed, the mean is fixed to zero $\mu=0$, and $\sigma^{2}$ is freely estimated so that the marginal likelihood is no 
longer dependent on $\theta$ (see Equation (4)). The integral in Equation (4) is approximated by, e.g., Gauss-Hermite quadrature by summing over a finite number of discrete quadrature points $\theta_{q}$ with $q=1,2, \ldots, Q$ and corresponding weights $w_{q}$ (see, e.g., Bock \& Aitkin, 1981; Bock \& Lieberman, 1970).

$$
L_{M M L}(\mathbf{X} \mid \boldsymbol{\beta}, \mathrm{G})=\prod_{x_{+}=0}^{I}\left[\exp \left(-\sum_{i=1}^{I} s_{i} \beta_{i}\right) \sum_{q=1}^{Q}\left(\frac{\exp \left(x_{+} \theta_{q}\right)}{\prod_{i=1}^{I}\left(1+\exp \left(\theta_{q}-\beta_{i}\right)\right)}\right) w_{q}\right]^{n_{x_{+}}}
$$

As Fischer (2007) stated, the marginal log-likelihood can be written as a sum of the conditional log-likelihood (9) and the marginal log-likelihood (6) of the raw-score frequencies as follows:

$$
\log L_{M M L}(\mathbf{X} \mid \boldsymbol{\beta}, \mathrm{G})=-\sum_{i=1}^{I} s_{i} \beta_{i}-\sum_{x_{+}=0}^{I} n_{x_{+}} \log \gamma\left(x_{+}, \beta\right)+\sum_{r} n_{x_{+}} \log \left(P\left(x_{+} \mid \boldsymbol{\beta}, \mathrm{G}\right)\right)
$$

The log-likelihood in the MML case in Equation (6) can be written as the sum of the CML part in Equation (9) and the marginalized log-likelihood frequencies of the raw scores (Fischer, 2007).

\subsection{Conditional Maximum Likelihood Estimation}

The parameter estimation process, initially proposed by Rasch, based on the idea of specific objectivity (simple sufficient statistics Rasch, 1960) is the CML estimation method (Andersen, 1972, 1973). The person parameter $\theta$ is eliminated from the likelihood by conditioning on the raw scores $x_{p+}$ as minimal sufficient statistic for the ability $\theta_{p}$ (Andersen, 1972, 1973; Rasch, 1960). Therefore, no distributional assumptions for the trait distribution have to be made. Thus, only the item parameters $\beta_{i}$, but not the person parameters $\theta_{p}$ are estimated. Person parameters have to be determined based on the estimated item parameters. For item parameter estimation, the elementary symmetric function $\gamma\left(x_{+}, \beta\right)$ of order $x_{+}$, is the crucial part of the likelihood in the estimation process. Different methods have been proposed for computing the elementary symmetric function (Formann, 1986; Verhelst, Glas, \& Van der Sluis, 1984), which differ in speed and accuracy. The likelihood of the response vector $\mathbf{x}_{+}=\left(x_{1+}, x_{2+}, \ldots, x_{P+}\right)$ can be written as:

$$
L\left(\mathbf{x}_{+} \mid \boldsymbol{\beta}\right)=\frac{\exp \left(\sum_{p=1}^{P} x_{p+} \theta_{p}\right) \prod_{p=1}^{P} \sum_{\mathbf{x}_{p i}}^{x_{p+}} \exp \left(-\sum_{i=1}^{I} x_{p i} \beta_{i}\right)}{\prod_{p=1}^{P} \prod_{i=1}^{I}\left(1+\exp \left(\theta_{p}-\beta_{i}\right)\right)}
$$


The likelihood in the CML case can then be written using Equations (3) and (7):

$$
L_{C M L}\left(\mathbf{X} \mid \mathbf{x}_{+}, \boldsymbol{\beta}\right)=\frac{L(\mathbf{X} \mid \boldsymbol{\theta}, \boldsymbol{\beta})}{L\left(\mathbf{x}_{+} \mid \boldsymbol{\beta}\right)}=\frac{\exp \left(-\sum_{i=1}^{I} s_{i} \beta_{i}\right)}{\prod_{x_{+}=0}^{I} \gamma\left(x_{+}, \boldsymbol{\beta}\right)^{n_{x_{+}}}}
$$

with $n_{x_{+}}$as the number of persons, who reached score $x_{+}$and $s_{i}=\sum_{i=1}^{I} x_{p i}$ as the item score. The log-likelihood is:

$$
\log L_{C M L}\left(\mathbf{X} \mid \mathbf{x}_{+}, \boldsymbol{\beta}\right)=-\sum_{i=1}^{I} s_{i} \beta_{i}-\sum_{x_{+}=0}^{I} n_{x_{+}} \log \gamma\left(x_{+}, \beta\right)
$$

As stated by Molenaar (1995), the resulting estimates $\hat{\boldsymbol{\beta}}$ are consistent as well as asymptotically efficient and asymptotically normally distributed.

\section{Parameter Estimation in MST Designs}

In recent years, the CML item parameter estimation in MST designs has been discussed. MST tests have design-related missing values; Mislevy and Sheehan (1989), referring to Rubin (1976), showed that MML provides consistent estimates in incomplete designs in general, and in MST designs, in particular (see also Wang et al., 2019). Following this justification, it can be shown, that MML can also be applied to MST designs (Eggen \& Verhelst, 2011; Wang et al., 2019; Zwitser \& Maris, 2015). In this context, it should be noted that the intuitively appropriate approach to assume that each of the routing paths in the MST can be represented as a group in a multiple group model, might not be ideal. As stated by Wang et al. (2019), this approach leads in any case to a bias in the parameter estimation.

As stated, the MML estimation requires a specification of a distribution $G$ for the usually unknown latent ability distribution. As Glas (1988) and also, for example, Zwitser and Maris (2015) argued, this assumption can be wrong, and the estimated parameters can be severely biased. In our experience, distributional misspecification has to be relatively large for MML estimation to perform worse compared to the CML parameter estimation (Steinfeld \& Robitzsch, 2021).

While the item parameters estimate in MML estimation is relatively unproblematic in MST designs, it is a challenge in CML estimation. Glas (1988) pointed out that the common CML item parameter estimation approach leads to severely biased estimates in MST designs (see also Eggen \& Verhelst, 2011; Kubinger, Steinfeld, Reif, \& Yanagida, 2012). On this result, it has been long recommended not to use the CML method especially because the MML estimation method offered an alternative (see also Steinfeld \& Robitzsch, 2021). In addition, in terms of the efficiency of item parameter estimates it is known that MML is superior to 
the CML estimation (Eggen \& Verhelst, 2006; Zwitser \& Maris, 2015). In applications in which it is difficult to assume distributional assumptions, the CML estimation could be yet advantageous. In addition to this benefit, supporters of the CML estimation method might also emphasize that this method comes close to the idea of person-free assessment (Molenaar, 1995) required for the postulation of specific objectivity (Rasch, 1977). Recently, the CML estimation in MST designs with deterministic routing could be solved while considering the respective design in the CML estimation process (Zwitser \& Maris, 2015). Here the symmetric function is modified, such that only those raw scores are considered, which can occur according to the respective MST design in each path. Considering an MST design with two stages (see Figure 1a), the possible raw scores are limited in the different paths of the MST design by the defined deterministic routing rules. As outlined in Section 2.2, the person parameter $\theta_{p}$ is eliminated from the likelihood (8), through conditioning on the raw score $x_{p+}$, without considering this limitation of possible raw scores. To apply the CML method also in MST designs, the routing rules must be integrated into the CML estimation (Zwitser \& Maris, 2015). Considering this approach, the probability of reaching a score of $X_{+}^{[1,2]}$ in the modules $\mathbf{m}^{[1,2]}$ with ability $\theta$, and the number of solved items in the module $\mathbf{m}^{[1]}$ being less than or equal to the cut score $c$ with $X_{+}^{[1]} \leq c$, can therefore be expressed as $P_{\mathbf{m}^{[1,2]}}\left(\mathbf{x}^{[1,2]} \mid \theta, X_{+}^{[1]} \leq c\right)=\frac{P_{\mathbf{m}^{[1,2]}}\left(\mathbf{x}^{[1,2]} \mid \theta\right)}{P_{\mathbf{m}^{[1,2]}}\left(X_{+}^{[1]} \leq c \mid \theta\right)}$. The elementary symmetric function introduced in Section 2.2 decomposed for one module results in $\gamma_{x_{+}}\left(\boldsymbol{m}^{[b]}\right)=\sum_{x: x_{+}^{[b]}=x_{+}} \prod_{i} \exp \left(-x_{i}^{[b]} \beta_{i}^{[b]}\right)$, with $\gamma_{x_{+}}\left(\boldsymbol{m}^{[b]}\right)=0$ if $x_{+}>I^{[b]}$, or $x_{+}<0$. Considering the routing rule e.g., $j \leq c$ for routing from module $\boldsymbol{m}^{[1]}$ to module $\boldsymbol{m}^{[2]}$ (path $\mathbf{m}^{[1,2]}$ ) and the associated limitation on possible raw scores, the probability of reaching a score $X_{+}^{[1,2]}$ considering these limitations can be expressed as $P_{\mathbf{m}^{[1,2]}}\left(\mathbf{x}^{[1,2]} \mid x_{+}^{[1,2]}, X_{+}^{[1]} \leq c\right)=\frac{\prod_{i} \exp \left(-x_{i}^{[1]} \beta_{i}^{[1]}\right) \prod_{j} \exp \left(-x_{j}^{[2]} \beta_{j}^{[2]}\right)}{\sum_{j=0}^{c} \gamma_{j}\left(\mathbf{m}^{[1]}\right) \gamma_{x_{+}^{[1,2]}-j}^{\left(\mathbf{m}^{[2]}\right)}}$.

With this modification of the common CML approach, item parameter estimation in deterministic MST designs is justified and leads to consistent item parameter estimates (Zwitser \& Maris, 2015). The proposed solution was implemented in the two R (R Core Team, 2020) packages dexterMST (Bechger, Koops, Partchev, \& Maris, 2019) and tmt (Steinfeld \& Robitzsch, 2019).

\subsection{Sequential Deterministic MST Designs with three Stages}

As stated in Section 1.1.1, MST designs can be assigned to those with sequential routing designs and cumulative routing designs. To make a differentiation between these designs, the previously described approach must first be extended.

Considering this approach for three stages (see Figure 1b), the probability of reaching a score of $X_{+}^{[1,2,3]}$ in the modules $\mathbf{m}^{[1,2,3]}$ with ability $\theta$, and the number of solved items in module $\mathbf{m}^{[1]}$ being less than or equal to the cut score $c_{1}$ and the number of solved items in 
module $\mathbf{m}^{[2]}$ being less than or equal to the cut score $c_{2}$ can be described as follows:

$$
\begin{aligned}
P_{\mathbf{m}^{[1,2,3]}}\left(\mathbf{x}^{[1,2,3]} \mid \theta, X_{+}^{[1]} \leq c_{1}, X_{+}^{[2]} \leq c_{2}\right) & =\frac{P_{\mathbf{m}^{[1,2,3]}}\left(\mathbf{x}^{[1,2,3]}, X_{+}^{[1]} \leq c_{1}, X_{+}^{[2]} \leq c_{2} \mid \theta\right)}{P_{\mathbf{m}^{[1,2,3]}}\left(X_{+}^{[1]} \leq c_{1}, X_{+}^{[2]} \leq c_{2} \mid \theta\right)} \\
& =\frac{P_{\mathbf{m}^{[1,2,3]}}\left(\mathbf{x}^{[1,2,3]} \mid \theta\right)}{P_{\mathbf{m}^{[1,2,3]}}\left(X_{+}^{[1]} \leq c_{1}, X_{+}^{[2]} \leq c_{2} \mid \theta\right)}
\end{aligned}
$$

Reformulating the symmetric function introduced in Section 3 with $\gamma_{x_{+}^{[1,2]} \mid x_{+}^{[1]} \leq c}\left(\mathbf{m}^{[1,2]}\right)=$ $\sum_{j=0}^{c} \gamma_{j}\left(\mathbf{m}^{[1]}\right) \gamma_{x_{+}^{[1,2]}-j}\left(\mathbf{m}^{[2]}\right)$ for three modules in sequential deterministic MST designs result in:

$$
\gamma_{x_{+}^{[1,2,3]} \mid x_{+}^{[1]} \leq c_{1}, x_{+}^{[2]} \leq c_{2}}\left(\mathbf{m}^{[1,2,3]}\right)=\sum_{j=0}^{c_{1}} \sum_{k=0}^{c_{2}} \gamma_{j}\left(\mathbf{m}^{[1]}\right) \gamma_{k}\left(\mathbf{m}^{[2]}\right) \gamma_{x_{+}^{[1,2,3]}-j-k}\left(\mathbf{m}^{[3]}\right)
$$

With this, the probability given the two deterministic cut scores $c_{1}$ and $c_{2}$, the probability of a score $X_{+}^{[1,2,3]}$ in sequential deterministic routing can be written as follows:

$$
\begin{aligned}
P_{\mathbf{m}^{[1,2,3]}}\left(\mathbf{x}^{[1,2,3]} \mid x_{+}^{[1,2,3]}, X_{+}^{[1]} \leq c_{1}, X_{+}^{[2]} \leq c_{2}\right) \\
=\frac{P_{\mathbf{m}^{[1,2,3]}}\left(\mathbf{x}^{[1,2,3]}, X_{+}^{[1]} \leq c_{1}, X_{+}^{[2]} \leq c_{2} \mid x_{+}^{[1,2,3]}\right)}{P_{\mathbf{m}^{[1,2,3]}}\left(X_{+}^{[1]} \leq c_{1}, X_{+}^{[2]} \leq c_{2} \mid x_{+}^{[1,2,3]}\right)} \\
=\frac{P_{\mathbf{m}^{[1,2,3]}}\left(\mathbf{x}^{[1,2,3]} \mid x_{+}^{[1,2,3]}\right)}{P_{\mathbf{m}^{[1,2,3]}}\left(X_{+}^{[1]} \leq c_{1}, X_{+}^{[2]} \leq c_{2} \mid x_{+}^{[1,2,3]}\right)} \\
=\frac{\prod_{i=1}^{I^{[1]}} \exp \left(-x_{i}^{[1]} \beta_{i}^{[1]}\right) \prod_{j=1}^{I^{[2]}} \exp \left(-x_{j}^{[2]} \beta_{j}^{[2]}\right) \prod_{k=1}^{I^{[3]}} \exp \left(-x_{k}^{[3]} \beta_{k}^{[3]}\right)}{\sum_{j=0}^{c_{1}} \sum_{k=0}^{c_{2}} \gamma_{j}\left(\mathbf{m}^{[1]}\right) \gamma_{k}\left(\mathbf{m}^{[2]}\right) \gamma_{x_{+}^{[1,2,3]}}-j-k}\left(\mathbf{m}^{[3]}\right)
\end{aligned}
$$

\subsection{Cumulative Deterministic MST Designs with three Stages}

In cumulative MST designs, all performance-related information from processed modules is taken into account for the routing. In MST designs with more than one routing decision, the given formulation needs an adjustment to cover these routing processes. Following the given illustration, the definition of the lower and upper bounds (which can be determined depending on the defined cut scores) need an adjustment as follows: 


$$
\begin{aligned}
P_{\mathbf{m}^{[1,2,3]}}\left(\mathbf{x}^{[1,2,3]} \mid \theta, X_{+}^{[1]} \leq c_{1}, X_{+}^{[1,2]} \leq c_{2}\right) \\
=\frac{P_{\mathbf{m}^{[1,2,3]}}\left(\mathbf{x}^{[1,2,3]}, X_{+}^{[1]} \leq c_{1}, X_{+}^{[1,2]} \leq c_{2} \mid \theta\right)}{P_{\mathbf{m}^{[1,2,3]}}\left(X_{+}^{[1]} \leq c_{1}, X_{+}^{[1,2]} \leq c_{2} \mid \theta\right)} \\
=\frac{P_{\mathbf{m}^{[1,2,3]}}\left(\mathbf{x}^{[1,2,3]} \mid \theta\right)}{P_{\mathbf{m}^{[1,2,3]}}\left(X_{+}^{[1]} \leq c_{1}, X_{+}^{[1,2]} \leq c_{2} \mid \theta\right)}
\end{aligned}
$$

In cumulative MST designs, the given cut score $c_{2}$, which includes the raw score of both module $\mathbf{m}^{[1,2]}$ has to be adjusted accordingly:

$$
\gamma_{x_{+}^{[1,2,3]} \mid x_{+}^{[1]} \leq c_{1}, x_{+}^{[1,2]} \leq c_{2}}\left(\mathbf{m}^{[1,2,3]}\right)=\sum_{j=0}^{c_{1}} \sum_{k=0}^{c_{2}-j} \gamma_{j}\left(\mathbf{m}^{[1]}\right) \gamma_{k}\left(\mathbf{m}^{[2]}\right) \gamma_{x_{+}^{[1,2,3]}-j-k}\left(\mathbf{m}^{[3]}\right)
$$

The probability of reaching a raw score $X_{+}^{[1,2,3]}$ conditioned, that the raw score in $X_{+}^{[1]}$ is not greater than $c_{1}$ and $X_{+}^{[1,2]}$ together is not greater than $c_{2}$ can be expressed as:

$$
\begin{aligned}
P_{\mathbf{m}^{[1,2,3]}}\left(\mathbf{x}^{[1,2,3]} \mid x_{+}^{[1,2,3]}, X_{+}^{[1]} \leq c_{1}, X_{+}^{[1,2]} \leq c_{2}\right) \\
=\frac{P_{\mathbf{m}^{[1,2,3]}}\left(\mathbf{x}^{[1,2,3]}, X_{+}^{[1]} \leq c_{1}, X_{+}^{[1,2]} \leq c_{2} \mid x_{+}^{[1,2,3]}\right)}{P_{\mathbf{m}^{[1,2,3]}}\left(X_{+}^{[1]} \leq c_{1}, X_{+}^{[1,2]} \leq c_{2} \mid x_{+}^{[1,2,3]}\right)} \\
=\frac{P_{\mathbf{m}^{[1,2,3]}}\left(\mathbf{x}^{[1,2,3]} \mid x_{+}^{[1,2,3]}\right)}{P_{\mathbf{m}^{[1,2,3]}}\left(X_{+}^{[1]} \leq c_{1}, X_{+}^{[1,2]} \leq c_{2} \mid x_{+}^{[1,2,3]}\right)} \\
=\frac{\prod_{i=1} \exp \left(-x_{i}^{[1]} \beta_{i}^{[1]}\right) \prod_{j=1}^{I^{[2]}} \exp \left(-x_{j}^{[2]} \beta_{j}^{[2]}\right) \prod_{k=1}^{I^{[3]}} \exp \left(-x_{k}^{[3]} \beta_{k}^{[3]}\right)}{\sum_{j=0}^{c_{1}} \sum_{k=0}^{c_{2}-j} \gamma_{j}\left(\mathbf{m}^{[1]}\right) \gamma_{k}\left(\mathbf{m}^{[2]}\right) \gamma_{x_{+}^{[1,2,3]}}-j-k}\left(\mathbf{m}^{[3]}\right)
\end{aligned}
$$

\section{Parameter Estimation in Probabilistic MST Designs}

In the following, the probabilistic routing as used for example in PIAAC (Yamamoto, Shin, et al., 2018), as well as in PISA (OECD, 2016) is introduced. In probabilistic routing, probabilities are defined by the test author for each raw score for the routing. The 
probabilities are chosen so that the routing to an easier module for persons with low raw scores (and therefore lower abilities) is greater than for a person with higher raw scores (or comparatively higher ability). With increasing ability usually, the probability also increases for the routing to a more difficult module (and vice versa).

The main difference between deterministic and probabilistic routing is that in the probabilistic case, there are normally no restrictions on possible raw scores (only if the routing probability for a specific raw score is zero). As the restriction of raw scores per path in the deterministic routing makes it necessary that the CML estimation method has to be modified, it is in the probabilistic routing the restriction based on the defined probabilities for each raw score in each MST path leading to another modification of the CML estimation approach. As stated, with the following modification both types of routing can be considered.

Following the modification of the common CML approach (see Section 2) for the deterministic routing as introduced in Section 3, let $\mathcal{C}_{2}$ be the event that the next module is chosen with score $X_{+}^{[1]}$. Here additionally $p_{2}(j)$ is introduced as the probability that based on the score $X_{+}^{[1]}$ a person will receive the next module $\mathbf{m}^{[2]}$. We are now changing the corresponding relationships from the previous section describing the deterministic routing, which will correspond to the special case $\mathcal{C}_{2}=\{j \leq c\}$.

$$
\begin{aligned}
P_{\mathbf{m}^{[1,2]}}\left(X_{+}^{[1]} \in \mathcal{C}_{2} \mid x_{+}^{[1,2]}\right) \\
=\frac{\sum_{j=0}^{I^{[1,2]}} p_{2}(j) \gamma_{j}\left(\mathbf{m}^{[1]}\right) \gamma_{x_{+}^{[1,2]}-j}\left(\mathbf{m}^{[2]}\right)}{\sum_{j=0}^{I^{[1,2]}} \gamma_{j}\left(\mathbf{m}^{[1]}\right) \gamma_{x_{+}^{[1,2]}-j}\left(\mathbf{m}^{[2]}\right)}
\end{aligned}
$$

It is further

$$
\begin{aligned}
P_{\mathbf{m}^{[1,2]}}\left(\mathbf{x}^{[1,2]} \mid x_{+}^{[1,2]}, X_{+}^{[1]} \in \mathcal{C}_{2}\right) & \\
= & \frac{P_{\mathbf{m}^{[1,2]}}\left(\mathbf{x}^{[1,2]}, X_{+}^{[1]} \in \mathcal{C}_{2} \mid x_{+}^{[1,2]}\right)}{P_{\mathbf{m}^{[1,2]}}\left(X_{+}^{[1]} \in \mathcal{C}_{2} \mid x_{+}^{[1,2]}\right)} \\
= & \frac{P_{\mathbf{m}^{[1,2]}}\left(\mathbf{x}^{[1,2]} \mid x_{+}^{[1,2]}\right)}{P_{\mathbf{m}^{[1,2]}}\left(X_{+}^{[1]} \in \mathcal{C}_{2} \mid x_{+}^{[1,2]}\right)}
\end{aligned}
$$




$$
=\frac{\prod_{i=1}^{I^{[1]}} \exp \left(-x_{i}^{[1]} \beta_{i}^{[1]}\right) \prod_{j=1}^{I^{[2]}} \exp \left(-x_{j}^{[2]} \beta_{j}^{[2]}\right)}{\sum_{j=0}^{[1,2]} p_{2}(j) \gamma_{j}\left(\mathbf{m}^{[1]}\right) \gamma_{x_{+}^{[1,2]}-j}\left(\mathbf{m}^{[2]}\right)}
$$

Equation (17) is the generalization of the deterministic routing introduced in Section 3 (Zwitser \& Maris, 2015). Here, the probabilities $p_{2}(j)$ are known in advance. In situations where the routing rules of the MST design are unknown and must be determined from the data, the probabilities $p_{2}$ are unknown as well. In these cases, they can be estimated by relative frequencies $\hat{p}_{2}(j)$, given that the next module is chosen if $X_{+}^{[1]}=j$. However, the modified CML estimate above is still consistent:

$$
P_{\mathbf{m}^{[1,2]}}\left(\mathbf{x}^{[1,2]} \mid x_{+}^{[1,2]}, X_{+}^{[1]} \leq c\right)=\frac{\prod_{i=1}^{I^{[1]}} \exp \left(-x_{i}^{[1]} \beta_{i}^{[1]}\right) \prod_{j=1}^{I^{[2]}} \exp \left(-x_{j}^{[2]} \beta_{j}^{[2]}\right)}{\sum_{j=0}^{I^{[1,2]}} p_{2}(j) \gamma_{j}\left(\mathbf{m}^{[1]}\right) \gamma_{x_{+}^{[1,2]}-j}\left(\mathbf{m}^{[2]}\right)}
$$

Since all $p_{2}(j)=1 \forall j \leq c, p_{2}(j)$ is canceled in the denominator. Furthermore, based on the definition of Zwitser and Maris (2015), we can use all $\gamma_{j}$ for which $j<0$ or $j$ larger the elements in $\mathbf{m}^{[b]}$ equals zero to rewrite Equation (17) as follows:

$$
P_{\mathbf{m}^{[1,2]}}\left(\mathbf{x}^{[1,2]} \mid x_{+}^{[1,2]}, X_{+}^{[1]} \leq c\right)=\frac{\prod_{i=1}^{I^{[1]}} \exp \left(-x_{i}^{[1]} \beta_{i}^{[1]}\right) \prod_{j=1}^{I^{[2]}} \exp \left(-x_{j}^{[2]} \beta_{j}^{[2]}\right)}{\sum_{j=0}^{c} \gamma_{j}\left(\mathbf{m}^{[1]}\right) \gamma_{x_{+}^{[1,2]}-j}\left(\mathbf{m}^{[2]}\right)}
$$

and thus get the deterministic version as it was formulated by Zwitser and Maris (2015). In summary, it can be seen that for the deterministic paths, more precisely the special case, described with $X_{+}^{[1]} \leq c, p_{2}(j)=0 \forall j>c$ the formulation proposed for probabilistic routing can be seen as a generalization of the approach proposed by Zwitser and Maris (2015).

It does not seem possible to make a CML estimate using the $P\left(\mathbf{x}^{[1,2]} \mid x_{+}^{[1]}, x_{+}^{[2]}\right)$ approach, due to the conditional independence.

$$
P\left(\mathbf{x}^{[1,2]} \mid x_{+}^{[1]}, x_{+}^{[2]}\right)=P\left(\mathbf{x}^{[1]} \mid x_{+}^{[1]}\right) P\left(\mathbf{x}^{[2]} \mid x_{+}^{[2]}\right)
$$

This means that the item parameters from the two modules are independent, and the parameters of the second module can only be identified up to a constant (empirically, the first parameter is set to zero, for example). Therefore it does not seem possible to map the 
item parameters of both modules on the same scale.

\subsection{Sequential Probabilistic MST Designs with three Stages}

As discussed in Section 3.1 as well as Section 3.2 in the following we will also formulate the approach for probabilistic routing separated for sequential and cumulative MST designs. For this purpose, it is again necessary to extend the MST design to at least one additional stage (see Figure 1b).

Considering this approach for three stages, the probability of reaching a score of $X_{+}^{[1,2,3]}$ in the modules $\mathbf{m}^{[1,2,3]}$ with ability $\theta$, based on the raw score $j$ in module $\mathbf{m}^{[1]}$ and $k$ in module $\mathbf{m}^{[2]}$ as well as the probability $p_{2}(j)$, and $p_{3}(k)$, can be written as follows. With $\mathcal{C}_{2}$ as the event that module $\mathbf{m}^{[2]}$ is chosen with the score $X_{+}^{[1]}$ and the probability $p_{2}(j)$. Let $\mathcal{C}_{3}$ be the event that module $\mathbf{m}^{[3]}$ is chosen with the score $X_{+}^{[2]}$ and the probability $p_{3}(k)$.

$$
\begin{aligned}
& P_{\mathbf{m}^{[1,2,3]}}\left(X_{+}^{[1]} \in \mathcal{C}_{2}, X_{+}^{[2]} \in \mathcal{C}_{3} \mid x_{+}^{[1,2,3]}\right) \sum_{j=0}^{I^{[1]}} \sum_{k=0}^{I^{[2]}} p_{2}(j) p_{3}(k) \gamma_{j}\left(\mathbf{m}^{[1]}\right) \gamma_{k}\left(\mathbf{m}^{[2]}\right) \gamma_{x_{+}^{[1,2,3]}-j-k}\left(\mathbf{m}^{[3]}\right) \\
& \sum_{j=0}^{I^{[1]}} \sum_{k=0}^{[2]} \gamma_{j}\left(\mathbf{m}^{[1]}\right) \gamma_{k}\left(\mathbf{m}^{[2]}\right) \gamma_{x_{+}^{[1,2,3]}-j-k}\left(\mathbf{m}^{[3]}\right)
\end{aligned}
$$

It is further

$$
\begin{aligned}
P_{\mathbf{m}^{[1,2,3]}}\left(\mathbf{x}^{[1,2,3]} \mid x_{+}^{[1,2,3]}, X_{+}^{[1]} \in \mathcal{C}_{2}, X_{+}^{[2]} \in \mathcal{C}_{3}\right) \\
=\frac{P_{\mathbf{m}^{[1,2,3]}}\left(\mathbf{x}^{[1,2,3]}, X_{+}^{[1]} \in \mathcal{C}_{2}, X_{+}^{[2]} \in \mathcal{C}_{3} \mid x_{+}^{[1,2,3]}\right)}{P_{\mathbf{m}^{[1,2,3]}}\left(X_{+}^{[1]} \in \mathcal{C}_{2}, X_{+}^{[2]} \in \mathcal{C}_{3} \mid x_{+}^{[1,2,3]}\right)} \\
=\frac{P_{\mathbf{m}^{[1,2,3]}}\left(\mathbf{x}^{[1,2,3]} \mid x_{+}^{[1,2,3]}\right)}{P_{\mathbf{m}^{[1,2,3]}}\left(X_{+}^{[1]} \in \mathcal{C}_{2}, X_{+}^{[2]} \in \mathcal{C}_{3} \mid x_{+}^{[1,2,3]}\right)} \\
=\frac{\prod_{i=1}^{I^{[1]}} \exp \left(-x_{i}^{[1]} \beta_{i}^{[1]}\right) \prod_{j=1}^{I^{[2]}} \exp \left(-x_{j}^{[2]} \beta_{j}^{[2]}\right) \prod_{k=1}^{I^{[3]}} \exp \left(-x_{k}^{[3]} \beta_{k}^{[3]}\right)}{\sum_{j=0}^{I^{[1]}} \sum_{k=0}^{I^{[2]}} p_{2}(j) p_{3}(k) \gamma_{j}\left(\mathbf{m}^{[1]}\right) \gamma_{k}\left(\mathbf{m}^{[2]}\right) \gamma_{x_{+}^{[1,2,3]}}-j-k}\left(\mathbf{m}^{[3]}\right)
\end{aligned}
$$




\subsection{Cumulative Probabilistic MST Designs with three Stages}

In the following, the cumulative probabilistic routing with three stages is illustrated. Persons are routed from the routing module to the second stage based on the score $X_{+}^{[1]}$ and the probability $p_{2}(j)$.

The further routing to the third stage deviates from that described in Section 3.1. Here the routing is based now on the raw score from both previously processed modules, like for example, $\mathbf{m}^{[1,2]}$, such that $\mathcal{C}_{3}$ describes the event, that module $\mathbf{m}^{[3]}$ is chosen based on the raw scores from $X_{+}^{[1,2]}$ and the probability $p_{3}(k)$ with $k$ including all possible raw scores of $\mathbf{m}^{[1,2]}$. Following this routing approach, the probability $P_{\mathbf{m}^{[1,2,3]}}\left(X_{+}^{[1]} \in \mathcal{C}_{2}, X_{+}^{[1,2]} \in \mathcal{C}_{3} \mid x_{+}^{[1,2,3]}\right)$ can be expressed as follows:

$$
\begin{aligned}
P_{\mathbf{m}}^{[1,2,3]}\left(X_{+}^{[1]} \in \mathcal{C}_{2}, X_{+}^{[1,2]} \in \mathcal{C}_{3} \mid x_{+}^{[1,2,3]}\right) \\
=\frac{\sum_{j=0}^{I^{[1]}} \sum_{k=0}^{I^{[2]}} p_{2}(j) p_{3}(j+k) \gamma_{j}\left(\mathbf{m}^{[1]}\right) \gamma_{k}\left(\mathbf{m}^{[2]}\right) \gamma_{x_{+}^{[1,2,3]}-j-k}\left(\mathbf{m}^{[3]}\right)}{\sum_{j=0}^{I^{[1]}} \sum_{k=0}^{I^{[2]}} \gamma_{j}\left(\mathbf{m}^{[1]}\right) \gamma_{k}\left(\mathbf{m}^{[2]}\right) \gamma_{x_{+}^{[1,2,3]}-j-k}\left(\mathbf{m}^{[3]}\right)}
\end{aligned}
$$

It is further

$$
\begin{aligned}
P_{\mathbf{m}^{[1,2,3]}}\left(\mathbf{x}^{[1,2,3]} \mid x_{+}^{[1,2,3]}, X_{+}^{[1]} \in \mathcal{C}_{2}, X_{+}^{[1,2]} \in \mathcal{C}_{3}\right) \\
=\frac{P_{\mathbf{m}^{[1,2,3]}}\left(\mathbf{x}^{[1,2,3]}, X_{+}^{[1]} \in \mathcal{C}_{2}, X_{+}^{[1,2]} \in \mathcal{C}_{3} \mid x_{+}^{[1,2,3]}\right)}{P_{\mathbf{m}^{[1,2,3]}}\left(X_{+}^{[1]} \in \mathcal{C}_{2}, X_{+}^{[1,2]} \in \mathcal{C}_{3} \mid x_{+}^{[1,2,3]}\right)} \\
=\frac{P_{\mathbf{m}^{[1,2,3]}}\left(\mathbf{x}^{[1,2,3]} \mid x_{+}^{[1,2,3]}\right)}{P_{\mathbf{m}^{[1,2,3]}}\left(X_{+}^{[1]} \in \mathcal{C}_{2}, X_{+}^{[1,2]} \in \mathcal{C}_{3} \mid x_{+}^{[1,2,3]}\right)} \\
=\frac{\prod_{i=1}^{[1]} \exp \left(-x_{i}^{[1]} \beta_{i}^{[1]}\right) \prod_{j=1}^{I^{[2]}} \exp \left(-x_{j}^{[2]} \beta_{j}^{[2]}\right) \prod_{k=1}^{I^{[3]}} \exp \left(-x_{k}^{[3]} \beta_{k}^{[3]}\right)}{\sum_{j=0}^{[1]} \sum_{k=0}^{I^{[2]}} p_{2}(j) p_{3}(j+k) \gamma_{j}\left(\mathbf{m}^{[1]}\right) \gamma_{k}\left(\mathbf{m}^{[2]}\right) \gamma_{x_{+}^{[1,2,3]}}-j-k}\left(\mathbf{m}^{[3]}\right)
\end{aligned}
$$




\section{Simulation Study}

\subsection{Method}

The simulation is based on the chosen parameters by Zwitser and Maris (2015) for simpler comparability (data generating parameters can be found here: https://osf.io/us6nd/?view_only=ac149eadd25141fbacea40d32b251987). We assume an MST with 50 items, which are composed of three modules $\mathbf{m}^{[1]}, \mathbf{m}^{[2]}, \mathbf{m}^{[3]}$ (see Figure 1a). Based on the raw score in the routing module $\mathbf{m}^{[1]}$, the examinees are routed either to $\mathbf{m}^{[2]}$ or $\mathbf{m}^{[3]}$. The routing module $\mathbf{m}^{[1]}$ includes 10 items, the other two modules $\mathbf{m}^{[2]}$ and $\mathbf{m}^{[3]}$ contain both 20 items. The item parameters for the first module $\mathbf{m}^{[1]}$ are drawn from a uniform distribution of $U(-1,1)$, those of $\mathbf{m}^{[2]}$ from $U(-2,-1)$, and the item parameters for the third module $\mathbf{m}^{[3]}$ are drawn from $U(1,2)$. The person parameters are drawn from a mixture of two normal distributions $\theta \sim \frac{1}{3} N(1,1)+\frac{2}{3} N(-1.5,0.5)$. For routing to the easier module $\mathbf{m}^{[2]}$, the weights $p_{1}=\{0.9,0.8,0.7,0.6,0.5,0.4,0.3,0.25 .0 .2,0.15,0.1\}$ were applied for the scores 0 to 10 . For the routing into the more difficult module $\mathbf{m}^{[3]}$, the weights $p_{2}=1-p_{1}$ were applied for the scores 0 to 10 . A person with a raw score of e.g. 0 in $\mathbf{m}^{[1]}$ is routed with a probability of $p_{1}=0.9$ to $\mathbf{m}^{[2]}$ (path 1 ) and $p_{2}=0.1$ to $\mathbf{m}^{[3]}$ (path 2).

For the simulation, six different sample sizes are considered $(N=$ $250,500,1000,3000,5000,10000)$. For each sample size, a total of 1,000 data sets were simulated and administered to the MST. The item parameters are estimated according to three different estimation methods. In addition to the probabilistic CML approach presented here, also the common CML approach without considering the MST design as well as an MML approach assuming that the person parameters are normally distributed are considered. To compare the estimated item parameters, all parameters were summed to zero. The simulation is conducted in $R$ ( $\mathrm{R}$ Core Team, 2020). For the CML estimation we used the package tmt (Steinfeld \& Robitzsch, 2019) in which we also implemented the modified CML approach for probabilistic MST designs (for deterministic routing, we received the same estimation results in a comparison of the two packages dexterMST and tmt). For the MML estimation, the package sirt (Robitzsch, 2020) was used.

The bias (BIAS) and root mean squared error (RMSE) is calculated for each condition and each module separately. The results are shown in Table 1 for all conditions and different sample sizes. The bias was calculated as the mean difference between the estimated parameter $\hat{\beta}_{i}$ and the true parameter $\beta_{i}$. The RMSE is the root of the squared difference between $\hat{\beta}_{i}$ and $\beta_{i}$ and represents a measure of the overall accuracy due to the combination of variability and bias. 


\section{$5.2 \quad$ Results}

The results of the simulation are summarized in Table 1. The BIAS, as well as the RMSE, are reported for each module and sample size $N$ rowise. Module 1 is the routing module. Module 2 is the module with easier and module 3 with more difficult items (see also Figure 1a). The column PCML contains the results for the modified CML method as introduced in Section 4. MML means the MML method with an assumed normal distribution of the person parameter $\theta$ (introduced in Section 2.1). The column CML contains the results for the common CML method without consideration of the MST design as described in Section 2.2.

Table 1

Bias (BIAS) and root mean squared error (RMSE) as a function of modules and sample sizes for all estimation methods

\begin{tabular}{|c|c|c|c|c|c|c|c|}
\hline \multirow[b]{2}{*}{$N$} & \multirow[b]{2}{*}{ module } & \multicolumn{3}{|c|}{ BIAS } & \multicolumn{3}{|c|}{ RMSE } \\
\hline & & PCML & MML & CML & PCML & MML & CML \\
\hline \multirow{3}{*}{250} & module 2 & -0.016 & 0.012 & -0.179 & 0.196 & 0.201 & 0.265 \\
\hline & module 1 & 0.000 & 0.031 & 0.003 & 0.164 & 0.163 & 0.168 \\
\hline & module 3 & 0.016 & -0.027 & 0.178 & 0.267 & 0.259 & 0.318 \\
\hline \multirow{3}{*}{500} & module 2 & -0.008 & 0.019 & -0.171 & 0.137 & 0.141 & 0.218 \\
\hline & module 1 & -0.003 & 0.029 & 0.000 & 0.114 & 0.116 & 0.117 \\
\hline & module 3 & 0.009 & -0.034 & 0.171 & 0.188 & 0.184 & 0.252 \\
\hline \multirow{3}{*}{1000} & module 2 & -0.005 & 0.022 & -0.168 & 0.097 & 0.102 & 0.194 \\
\hline & module 1 & -0.002 & 0.030 & 0.001 & 0.081 & 0.085 & 0.083 \\
\hline & module 3 & 0.006 & -0.036 & 0.168 & 0.132 & 0.133 & 0.212 \\
\hline \multirow{3}{*}{3000} & module 2 & -0.003 & 0.024 & -0.166 & 0.068 & 0.074 & 0.179 \\
\hline & module 1 & -0.001 & 0.031 & 0.002 & 0.057 & 0.066 & 0.060 \\
\hline & module 3 & 0.003 & -0.039 & 0.165 & 0.093 & 0.099 & 0.189 \\
\hline \multirow{3}{*}{5000} & module 2 & -0.001 & 0.025 & -0.164 & 0.043 & 0.052 & 0.169 \\
\hline & module 1 & 0.000 & 0.032 & 0.003 & 0.036 & 0.050 & 0.039 \\
\hline & module 3 & 0.001 & -0.041 & 0.163 & 0.059 & 0.071 & 0.173 \\
\hline \multirow{3}{*}{10000} & module 2 & 0.000 & 0.026 & -0.164 & 0.030 & 0.042 & 0.166 \\
\hline & module 1 & 0.000 & 0.032 & 0.003 & 0.025 & 0.043 & 0.029 \\
\hline & module 3 & 0.000 & -0.042 & 0.162 & 0.041 & 0.059 & 0.167 \\
\hline
\end{tabular}

Note. $\quad N=$ sample size; PCML $=$ probabilistic CML; MML with an assumed normal distribution of $\theta ; \mathrm{CML}=$ conventional $\mathrm{CML}$ without consideration of the MST design. The modules are sorted after mean difficulty. Module 1 is the routing module, module 2 those with easier, and module 3 with more difficult items. 
Concerning the bias of the item parameter estimates across all sample sizes the results indicate that the modified CML method (column PCML) leads to almost unbiased item parameter estimates, even in the condition with comparatively small sample sizes of $N=250$. Both the MML estimation and the common CML method (column MML and CML) lead to a bias of the item parameter estimates. Even with larger sample sizes (e.g. $N=5000,10000)$ the BIAS did not vanish. These results are to be expected. The common CML method does not consider the MST design and the corresponding raw score restrictions and the applied MML estimation assumed (wrongly) a normal distribution of the person parameter. As already noted, the MML method is also applicable in MST designs and the BIAS here reflects the misspecified distribution of the person parameter. These results are similar to those reported for deterministic MST designs (Zwitser \& Maris, 2015). It should be emphasized that the BIAS for the MML method is comparatively small, although the underlying distribution of the person parameter is misspecified (see also Section 3 or Steinfeld and Robitzsch (2021) for a detailed discussion on parameter estimation in MST designs under different trait distributions). In terms of the RMSE, the results for the BIAS are supported, which becomes clear especially with increasing sample sizes $(N>1000)$. The RMSE for the modified CML method performed best and lies between [0.267, 0.041] for module 3 , the module with the most difficult items, and the highest RMSE. Regarding the MML method, the RMSE is comparable to those of the modified CML method and ranges between [0.259, 0.059], also for module 3. The RMSE is the largest for the common CML method with $[0.318,0.167]$ for module 3 and also did not decreases much for larger sample sizes. As expected for the routing module (module 1), the RMSE for the common CML method is comparable to that of the modified CML method (PCML). This is expected as here the raw scores are not restricted.

\section{Summary and Discussion}

This article introduces a modified CML estimation approach for probabilistic MST designs. In the past, few contributions have dealt with the CML estimation in MST designs and here only for deterministic MST designs. As stated by Glas (1988) the application of the common CML method in MST designs leads to severely biased item parameter estimates. It was then recommended not to apply the common CML method to calibrate all item parameters but to apply the method for each path in the MST design separately. This is not a satisfactory option because the estimated item parameters cannot be aligned on one common scale. Zwitser and Maris (2015) introduced a modified CML method for deterministic MST designs and were able to show that taking the respective MST design into account leads to unbiased item parameters. As indicated, the proposed solution is only applicable for deterministic MST designs. Deterministic MST designs are characterized by routing based 
on a cut score $c$ for the raw score $j$ in a module $\mathbf{m}$ as well as a rule such as $j \leq c$ for the routing to an easier module. This type of routing means that not all raw scores can occur in all paths of the MST design, which must be taken into account in the CML item parameter estimation approach Zwitser and Maris (2015) (due to conditioning on the raw score; see Section 2.2). In probabilistic MST designs, as used in international large-scale assessments, like PIAAC and PISA, the proposed modification for the CML estimation is not applicable. Instead of a cut score $c$ for all raw scores $j$ in module $\mathbf{m}$ and the associated restriction on possible raw scores per path, the routing is based on a probability $p(j)$ defined for each raw score $j$. Therefore all raw scores are theoretically possible but are restricted by the specified probability, which must be taken into account in the CML estimation. Accordingly, the common CML approach is again modified to also take probabilistic MST into account. It is important to notice, that with the proposed modification the item parameters for both the deterministic as well as the probabilistic MST designs can be estimated. It could be shown in a simulation study with different sample sizes and different estimation methods that the proposed modification of the common CML approach for probabilistic MST designs leads to consistent item parameter estimates, compared to the common CML estimation method. At this point, it should be emphasized, that the MML estimation does not perform significantly worse, even with a major misspecified distribution of the person parameters. The approach presented here for CML estimation in probabilistic designs is limited to the 1PL but could be extended to the partial credit model (PCM; Masters, 1982).

\section{References}

Andersen, E. B. (1972). The numerical solution of a set of conditional estimation equations. Journal of the Royal Statistical Society: Series B (Methodological), 34(1), 42-54. https://doi.org/10.1111/j.2517-6161.1972.tb00887.x

Andersen, E. B. (1973). Conditional inference and models for measuring. Denmark: Mentalhygiejnisk Forlag.

Baker, F. B., \& Kim, S.-H. (2004). Item Response Theory: Parameter estimation techniques. New York: Dekker.

Bechger, T., Koops, J., Partchev, I., \& Maris, G. (2019). dexterMST: CML Calibration of Multi Stage Tests [R Package Version 0.1.2; R Core Team: Vienna, Austria, 2020; Available online: https://CRAN.R-project.org/package=dexterMST (accessed on 03 April 2020)].

Betz, N. E., \& Weiss, D. J. (1974). Simulation studies of two-stage ability testing (Research Report No. 74-4). Psychometric Methods Program, Department of Psychology, University of Minnesota, Minneapolis.

Bock, R. D., \& Aitkin, M. (1981). Marginal maximum likelihood estimation of item parameters: Application of an EM algorithm. Psychometrika, 46(4), 443-459. https: //doi.org/10.1007/bf02293801 
Bock, R. D., \& Lieberman, M. (1970). Fitting a response model for n dichotomously scored items. Psychometrika, 35(2), 179-197. https://doi.org/10.1007/BF02291262

Campbell, J. R., Hombo, C. M., \& Mazzeo, J. (2000). NAEP 1999 trends in academic progress: Three decades of student performance. ERIC.

Chang, H.-H. (2015). Psychometrics behind computerized adaptive testing. Psychometrika, 80(1), 1-20. https://doi.org/10.1007/s11336-014-9401-5

Chen, H., Yamamoto, K., \& von Davier, M. (2014). Controlling multistage testing exposure rates in international large-scale assessments. In A. Yan, A. A. von Davier, \& C. Lewis (Eds.), Computerized multistage testing: Theory and applications (pp. 391-409). New York: CRC Press. https://doi.org/10.1201/b16858

Cronbach, L. J., \& Gleser, G. C. (1957). Psychological tests and personnel decisions. Urbana: University of Illinois Press.

De Boeck, P. (2008). Random item IRT models. Psychometrika, 73(4), 533. https://doi.org/ 10.1007/s11336-008-9092-x

Drasgow, F., Luecht, R. M., \& Bennett, R. E. (2006). Technology and testing. In R. Bennett (Ed.), Educational measurement (4th ed., pp. 471-515). Westport, CT: American Council on Education/Praeger.

Eggen, T. J. H. M., \& Verhelst, N. D. (2006). Loss of information in estimating item parameters in incomplete designs. Psychometrika, 71 (2), 303-322. https://doi.org/ 10.1007/s11336-004-1205-6

Eggen, T. J. H. M., \& Verhelst, N. D. (2011). Item calibration in incomplete testing designs. Psicologica: International Journal of Methodology and Experimental Psychology, 32(1), 107-132.

Fischer, G. H. (2007). Rasch models. In C. R. Rao \& S. Sinharay (Eds.), Handbook of statistics, volume 26: Psychometrics (pp. 515-585). North Holland: Elsevier. https: //doi.org/10.1016/S0169-7161(06)26016-4

Fishbein, B., Martin, M. O., Mullis, I. V., \& Foy, P. (2018). The TIMSS 2019 Item Equivalence Study: Examining mode effects for computer-based assessment and implications for measuring trends. Large-scale Assessments in Education, 6(1), 1-23. https: //doi.org/10.1186/s40536-018-0064-z

Formann, A. K. (1986). A note on the computation of the second-order derivatives of the elementary symmetric functions in the Rasch model. Psychometrika, 51(2), 335-339. https://doi.org/10.1007/BF02293990

Glas, C. A. W. (1988). The Rasch model and multistage testing. Journal of Educational Statistics, 13(1), 45-52. https://doi.org/10.2307/1164950

Hendrickson, A. (2007). An NCME instructional module on multistage testing. Educational Measurement: Issues and Practice, 26(2), 44-52. https://doi.org/10.1111/j.17453992.2007.00093.x

Holland, P. W. (1990). On the sampling theory roundations of item response theory models. Psychometrika, 55(4), 577-601. https://doi.org/10.1007/BF02294609

Jodoin, M. G., Zenisky, A., \& Hambleton, R. K. (2006). Comparison of the psychometric properties of several computer-based test designs for credentialing exams with multiple purposes. Applied Measurement in Education, 19(3), 203-220. https://doi. org/10.1207/s15324818ame1903_3 
Kim, H., \& Plake, B. S. (1993). Monte carlo simulation comparison of two-stage testing and computerized adaptive testing [Paper presented at the annual meeting of the National Council on Measurement in Education, Atlanta, GA].

Kim, S., Moses, T., \& Yoo, H. H. (2015). Effectiveness of item response theory (IRT) proficiency estimation methods under adaptive multistage testing. ETS Research Report Series, 2015(1), 1-19. https://doi.org/10.1002/ets2.12057

Kubinger, K., \& Holocher-Ertl. (2014). AID 3: Adaptives Intelligenz Diagnostikum 3 [AID 3: Adaptive Intelligence Diagnostic 3]. Göttingen: Beltz-Test.

Kubinger, K. D., Steinfeld, J., Reif, M., \& Yanagida, T. (2012). Biased (conditional) parameter estimation of a Rasch model calibrated item pool administered according to a branched testing design. Psychological Test and Assessment Modeling, 52(4), 450-460.

Lord, F. M. (1971). A theoretical study of two-stage testing. Psychometrika, 36(3), 227-242. https://doi.org/10.1007/BF02297844

Lord, F. M. (1980). Applications of Item Response Theory to practical testing problems. Hillsdale, NJ: Erlbaum. https://doi.org/10.4324/9780203056615

Lord, F. M., Novick, M. R., \& Birnbaum, A. (1968). Statistical theories of mental test scores. Menlo Park: Addison-Wesley.

Luecht, R. M., \& Nungester, R. J. (1998). Some practical examples of computer-adaptive sequential testing. Journal of Educational Measurement, 35(3), 229-249. https: //doi.org/10.1111/j.1745-3984.1998.tb00537.x

Magis, D., Yan, D., \& von Davier, A. A. (2017). Computerized adaptive and multistage testing with R: Using packages catR and mstR. Cham: Springer. https://doi.org/10.1007/9783-319-69218-0

Masters, G. N. (1982). A Rasch model for partial credit scoring. Psychometrika, 47(2), $149-174$.

Mislevy, R. J., \& Sheehan, K. M. (1989). The role of collateral information about examinees in item parameter estimation. Psychometrika, 54 (4), 661-679. https://doi.org/10. 1007/BF02296402

Molenaar, I. W. (1995). Some background for item response theory and the Rasch model. In G. H. Fischer \& I. Molenaar (Eds.), Rasch models, recent developments and applications (pp. 3-14). New York: Springer. https://doi.org/10.1007/978-1-46124230-7

Mullis, I., \& Martin, M. O. (2019). PIRLS 2021 assessment frameworks [Retrieved from Boston College, TIMSS \& PIRLS International Study Center website: https:// timssandpirls.bc.edu/pirls2021/frameworks/].

Organisation for Economic Co-operation and Development. (2010). "PISA computer-based assessment of student skills in science". OECD Publishing. https://doi.org/10.1787/ 9789264082038-en

Organisation for Economic Co-operation and Development. (2016). PISA 2018 integrated design (tech. rep.). OECD Publishing. https://www.oecd.org/pisa/pisaproducts/ PISA-2018-INTEGRATED-DESIGN.pdf

Organisation for Economic Co-operation and Development. (2019a). PISA 2018 assessment and analytical framework. OECD Publishing. https://doi.org/10.1787/b25efab8-en 
Organisation for Economic Co-operation and Development. (2019b). Technical report of the survey of adult skills (PIAAC) (third edition) (2019). OECD Publishing.

$\mathrm{R}$ Core Team. (2020). R: A language and environment for statistical computing [R Core Team: Vienna, Austria, 2020; Available online: https://www.R-project.org/ (accessed on 1 February 2020)].

Rasch, G. (1960). Probabilistic models for some intelligence and attainment tests. Copenhagen, Denmark: Pædagogiske Institut.

Rasch, G. (1977). On specific objectivity. An attempt at formalizing the request for generality and validity of scientific statements. In M. Blegvad (Ed.), The Danish year-book of philosophy (pp. 58-94). Copenhagen: Munksgaard.

Robitzsch, A. (2020). sirt: Supplementary Item Response Theory Models [R Package Version 3.9-4; R Core Team: Vienna, Austria, 2020; Available online: https://CRAN.Rproject.org/package $=$ sirt $($ accessed on 03 April 2020)].

Rubin, D. B. (1976). Inference and missing data. Biometrika, 63(3), 581-592. https://doi. org/10.1093/biomet/63.3.581

San Martin, E., \& De Boeck, P. (2015). What do you mean by a difficult item? On the interpretation of the difficulty parameter in a Rasch model. In R. E. Millsap, D. M. Bolt, L. A. van der Ark, \& W.-C. Wang (Eds.), Quantitative psychology research. The 78th annual meeting of the psychometric society, springer proceedings in mathematics E statistics (pp. 1-14). New York: Springer. https://doi.org/10.1007/978-3-31907503-7

Steinfeld, J., \& Robitzsch, A. (2019). tmt: Estimation of the Rasch model for multistage tests [R Package Version 0.2.1-0; R Core Team: Vienna, Austria, 2020; Available online: https://CRAN.R-project.org/package=tmt (accessed on 03 April 2020)].

Steinfeld, J., \& Robitzsch, A. (2021). Item parameter estimation in multistage designs: A comparison of different estimation approaches for the Rasch model. Psych, 3(3), 279-307. https://doi.org/10.3390/psych3030022

Svetina, D., Liaw, Y.-L., Rutkowski, L., \& Rutkowski, D. (2019). Routing strategies and optimizing design for multistage testing in international large-scale assessments. Journal of Educational Measurement, 56(1), 192-213. https://doi.org/10.1111/jedm. 12206

Thissen, D. (1982). Marginal maximum likelihood estimation for the one-parameter logistic model. Psychometrika, 47(2), 175-186. https://doi.org/10.1007/BF02296273

van der Linden, W. J. (2005). Linear models for optimal test design. New York: Springer. https://doi.org/10.1007/0-387-29054-0

van der Linden, W. J., \& Glas, C. A. (2010). Elements of Adaptive Testing. New York: Springer. https://doi.org/10.1007/978-0-387-85461-8

Verhelst, N. D., Glas, C., \& Van der Sluis, A. (1984). Estimation problems in the Rasch model: The basic symmetric functions. Computational Statistics Quarterly, 1(3), $245-262$.

Wainer, H., Dorans, N. J., Flaugher, R., Green, B. F., Mislevy, R. J., Steinberg, L., \& Thissen, D. (2000). Computerized adaptive testing: A primer (2. ed.). Lawrence Erlbaum. 
Wang, C., Chen, P., \& Jiang, S. (2019). Item calibration methods with multiple subscale multistage testing. Journal of Educational Measurement. https://doi.org/10.1111/ jedm.12241

Weiss, D. J. (1982). Improving measurement quality and efficiency with adaptive testing. Applied Psychological Measurement, 6(4), 473-492. https://doi.org/10.1177\% 2F014662168200600408

Weiss, D. J. (1983). New Horizons in Testing. New York: Academic Press. https://doi.org/ 10.1016/C2009-0-03014-1

Weiss, D. J., \& Kingsbury, G. G. (1984). Application of computerized adaptive testing to educational problems. Journal of Educational Measurement, 21(4), 361-375. https://doi.org/10.1111/j.1745-3984.1984.tb01040.x

Yamamoto, K., \& Khorramdel, L. (2018). Introducing multistage adaptive testing into international large-scale assessments designs using the example of PIAAC. Psychological Test and Assessment Modeling, 60(3), 347-368.

Yamamoto, K., Shin, H. J., \& Khorramdel, L. (2018). Multistage adaptive testing design in international large-scale assessments. Educational Measurement: Issues and Practice, 37(4), 16-27. https://doi.org/10.1111/emip.12226

Yamamoto, K., Shin, H. J., \& Khorramdel, L. (2019). Introduction of multistage adaptive testing design in PISA 2018 (OECD Education Working Paper No. 209). https: //doi.org/10.1787/b9435d4b-en

Zenisky, A., Hambleton, R. K., \& Luecht, R. M. (2009). Multistage testing: Issues, designs, and research. In W. J. van der Linden \& C. A. Glas (Eds.), Elements of adaptive testing (pp. 355-372). New York: Springer. https://doi.org/10.1007/978-0-387-854618

Zhang, T., Xie, Q., Park, B. J., Kim, Y. Y., Broer, M., \& Bohrnstedt, G. (2016). Computer familiarity and its relationship to performance in three NAEP digital-based assessments. AIR-NAEP Working Paper\# 01-2016. American Institutes for Reasearch.

Zwitser, R. J., \& Maris, G. (2015). Conditional statistical inference with multistage testing designs. Psychometrika, 80(1), 65-84. https://doi.org/10.1007/s11336-013-9369-6 